\title{
An updated diced cartilage fascia technique for dorsal augmentation in rhinoplasty
}

\author{
Jinli Wu', Donald B. Yoo ${ }^{1,2,3}$ \\ 'Private practice, Facial Plastic \& Reconstructive Surgery, Beverly Hills, CA 90212, USA. \\ ${ }^{2}$ Division of Facial Plastic \& Reconstructive Surgery, Department of Otolaryngology - Head \& Neck Surgery, University of Southern \\ California, Los Angeles, CA 90033, USA. \\ ${ }^{3}$ Division of Facial Plastic \& Reconstructive Surgery, Department of Otolaryngology - Head \& Neck Surgery, University of California - Los \\ Angeles, Los Angeles, CA 90095, USA.
}

Correspondence to: Dr. Donald B. Yoo, Facial Plastic Surgery, 120 S. Spalding Drive Suite 303, Beverly Hills, CA 90212, USA.

E-mail: info@donyoomd.com

How to cite this article: Wu J, Yoo DB. An updated diced cartilage fascia technique for dorsal augmentation in rhinoplasty. Plast Aesthet Res 2019;6:5. http://dx.doi.org/10.20517/2347-9264.2018.74

Received: 24 Oct 2018 First Decision: 30 Oct 2018 Revised: 21 Dec 2018 Accepted: 29 Jan 2019 Published: 22 Mar 2019

Science Editor: Hong Ryul Jin Copy Editor: Cai-Hong Wang Production Editor: Huan-Liang Wu

\begin{abstract}
The aim of this paper is to discuss an updated technique for dorsal augmentation during rhinoplasty using diced cartilage wrapped in fascia. The usage of diced cartilage has been variously described in the literature with consistently satisfactory results. Herein, we present our experience with patients undergoing dorsal augmentation during rhinoplasty using an updated method of diced cartilage wrapped in fascia. Diced cartilage fascia techniques have become the technique of choice for dorsal augmentation for an ever-increasing number of rhinoplasty surgeons. The term is broadly descriptive and there remains a wide-range of ways to execute. Updating and enhancing the technique with greater attention to precision, and creating an aesthetically optimal and predictable result, may result in even improved outcomes for future patients.
\end{abstract}

Keywords: Rhinoplasty, revision rhinoplasty, dorsal augmentation, costal cartilage, diced cartilage, asian rhinoplasty, DCF, diced cartilage fascia

\section{INTRODUCTION}

In the practice of medicine, the concept of the "gold standard" refers to the best available test or treatment under reasonable conditions. Given the relative lack of purely objective experimentation and testing in

CC (7) (c) The Author(s) 2019. Open Access This article is licensed under a Creative Commons Attribution 4.0 International License (https://creativecommons.org/licenses/by/4.0/), which permits unrestricted use sharing, adaptation, distribution and reproduction in any medium or format, for any purpose, even commercially, as long as you give appropriate credit to the original author(s) and the source, provide a link to the Creative Commons license, and indicate if changes were made. 
rhinoplasty, no such criterion standard yet exists for dorsal augmentation. In the ongoing pursuit of the optimal technique for augmenting the dorsum during primary and revision rhinoplasty, surgeons have continuously sought to increase precision, safety, and permanence.

The history of dorsal augmentation during rhinoplasty emulates in many ways the progression of increasingly higher standards of care in medicine driven by technological advances and rapidly evolving therapies. Early attempts were decidedly crude, with a wide assortment of everyday materials including ivory ${ }^{[1]}$ and jade used to increase the height of the nose. Through the years surgeons have attempted to improve outcomes by utilizing a variety of autologous and alloplastic materials, including: cartilage, bone ${ }^{[2-4]}$, fascia $^{[5]}$ diced cartilage and fascia ${ }^{[6-9]}$, silicone ${ }^{[10-12]}$, medpore ${ }^{[13]}$, polytetrafluorethylene ${ }^{[14,15]}$, supramid ${ }^{[16]}$, proplast $^{[17]}$, vicryl ${ }^{[18]}$, and mersilene ${ }^{[19]}$. All with mixed results.

While many contemporary surgeons favor autologous grafts in an onlay configuration for mild to moderate amounts of dorsal augmentations ${ }^{[2,10,20]}$, cases demanding a larger volume of graft materials have prompted surgeons to explore alloplastic (silicone, Goretex, etc.) and homoplastic (irradiated costal cartilage) options in addition to autologous options given the ease of obtaining grafts, and the absence of any donor site morbidity ${ }^{[2-5]}$. However, a primary downside of these grafts has proven to be the relatively high risk of complications compared to autologous graft techniques, driving other surgeons to pursue this avenue more intently.

The use of diced cartilage in dorsal augmentation has been periodically documented in the Englishlanguage literature as early as 1943 by Peer, in 1951 by Cottle, and in 1968 by Burian, though it did not gain wide-spread acceptance at the time ${ }^{[21-23]}$. Guerrerosantos revisited this concept in the $1990 \mathrm{~s}^{[8]}$, refining the technique by wrapping fragmented cartilage in fascia, while Erol brought a larger audience with his description of wrapping diced cartilage in Surgicel in $2000^{[24]}$, then Daniel subsequently brought a renewed interested in wrapping diced cartilage in fascia ${ }^{[6,7]}$. Modifications of the concept of using diced cartilage as the building block for dorsal augmentation have been variously described, primarily adding assorted tissue adhesives to ease shaping of the graft, altering the material wrapping the cartilage, or foregoing an encasement altogether ${ }^{[9,25-30]}$. The manifold existing descriptions in the literature notwithstanding, a systematic approach refining the surgical technique to achieve greater precision and consistency using diced cartilage with fascia has not been previously delineated.

Diced cartilage with fascia represents a potentially ideal graft for dorsal augmentation as it makes use of the lower complication rates associated with autologous grafts, while also providing a graft that has the ability to recreate dorsal aesthetic lines in a natural and predictable manner. The usage of diced cartilage has been variously described in the literature, with consistently satisfactory results reported. Herein, we present our experience, with patients undergoing dorsal augmentation during rhinoplasty, using an updated method of diced cartilage wrapped in fascia.

\section{SURGICAL TECHNIQUE}

Proper surgical planning and preparation for dorsal augmentation begins with the consultation and preoperative visit, wherein the nasal anatomy should be thoroughly assessed, and the aesthetic goals of surgery defined, with particular attention directed at the dorsum, established with the patient.

The primary consideration with regards to the pre-operative nasal anatomy is the shape and integrity of the platform created by the confluence of the upper lateral cartilages along the dorsal septum. The presence of significant contour irregularities such as a dorsal hump or inverted-V deformities, indicate the need for proper preparation and modification of the dorsum to support a diced cartilage wrapped in fascia (DCF) 


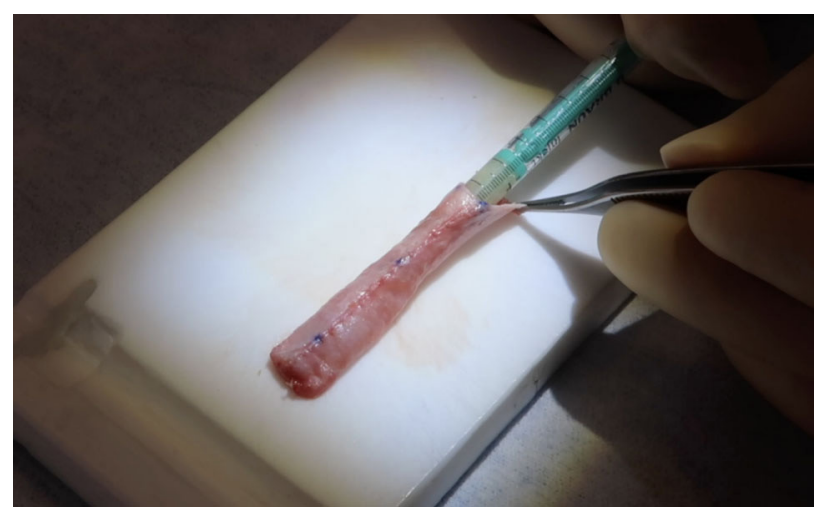

Figure 1. Care is taken to ensure the diced cartilage flows freely through the hub of the syringe

graft. Physical exam findings in conjunction with the patient's aesthetic desires dictate the most appropriate source of graft material.

Computer-imaging is also a beneficial communication tool between surgeon and patient as it allows a focused discussion of the patient's anticipated results with the realities and limitations of surgery, as well as the types and degrees of potential changes. This provides the surgeon an opportunity to more accurately gauge the desired shape of the nose and dorsum with regards to nasofrontal angle, radix height, dorsal height, length, and supratip break, which become important considerations in shaping the DCF.

Pre-operatively, the patient is marked in the upright position. The anticipated nasal starting point, dorsal convexity - if present, desired supratip break, and the midline of the face should be marked, as well as the inframammary/infrapectoral crease and xiphoid in the case of costal cartilage harvest.

Cartilage may be harvested from the septum, ears, or rib, depending on the volume requirements of the dorsal augmentation. The physical characteristics of the cartilage sources do vary, with softer cartilage allowing for finer dicing and greater pliability once placed within fascia. Dicing of the cartilage to $<0.5 \mathrm{~mm}$ pieces is recommended to minimize the risk of contour irregularities, as shown in Figure 1.

While fascia may be obtained from multiple sources, deep temporalis fascia is the thinnest of commonly used options, and produces minimal donor site morbidity. Once healed, the diced cartilage within the DCF provides the lasting volume, while the fascia simply acts as a temporary vehicle to place and shape the cartilage. For this reason, thinner fascia is preferable for more precise titration of graft size and shape. Care should be taken during fascia harvest to ensure adequate surface area $(>5 \mathrm{~cm} \times 3.5 \mathrm{~cm})$ and that all extraneous fat and muscle is meticulously removed to create the thinnest and most uniform layer of tissue, as shown in Figures 2 and 3.

Once the deep temporalis fascia has been thinned, it is sutured longitudinally into a cylindrical shape with a running-locking 5-0 vicryl to avoid any escape of diced cartilage from the construct. The width of the cylinder is determined by the desired width as well as height of the patient's bridge, generally in a range between 3.2-3.5 cm of fascia diameter. One end of the fascia is then closed and filled with an estimated volume of diced cartilage, then placed along the nasal dorsum.

The DCF will contract and dehydrate when healed, so every effort is made to remove fluid from the DCF prior to making measurements for its final dimensions. The cephalic end of the DCF is placed at the previously marked nasal starting point, and the supratip break marked caudally. The fascia is then closed 


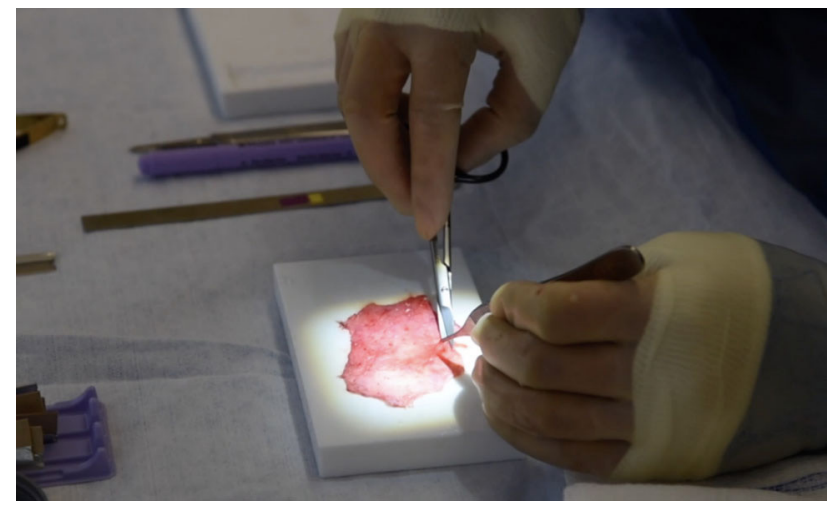

Figure 2. Removal of all excess fat, muscle, and adherent superficial fascia to preserve only the deep temporalis fascia

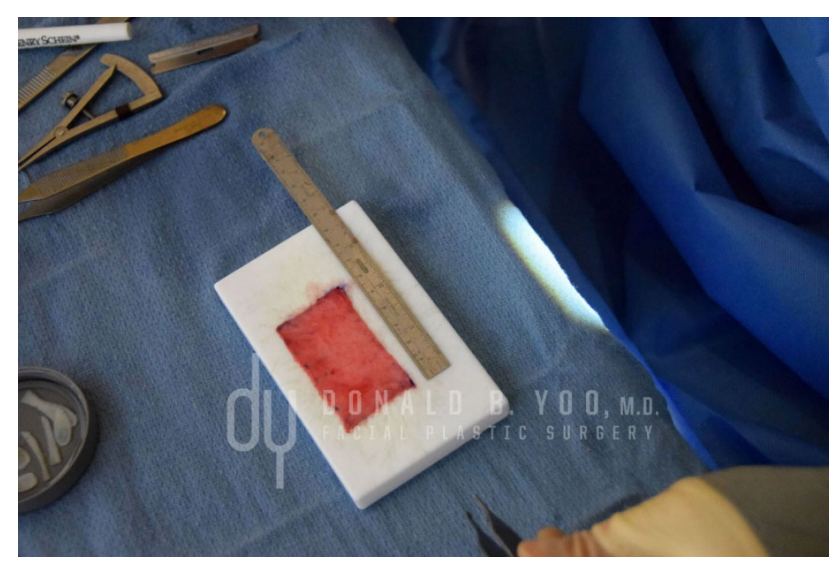

Figure 3. Demonstrating adequate fascia surface area $(>5 \mathrm{~cm} \times 3.5 \mathrm{~cm})$

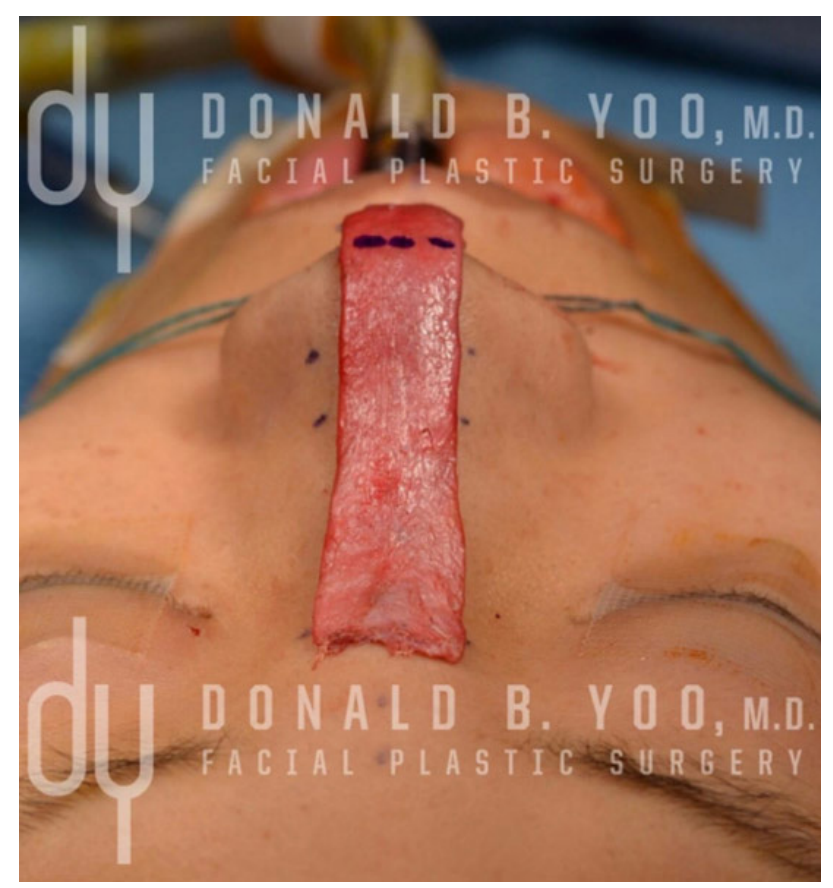

Figure 4. The dimensions of the diced cartilage wrapped in fascia are determined by placement on top of the patient's dorsum 


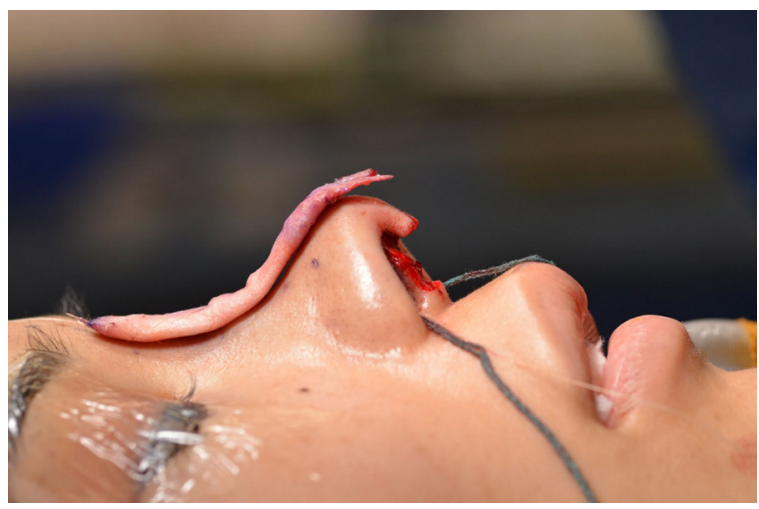

Figure 5. The diced cartilage wrapped in fascia is adjusted to account for the nasal starting point, dorsal aesthetic line, and supratip break

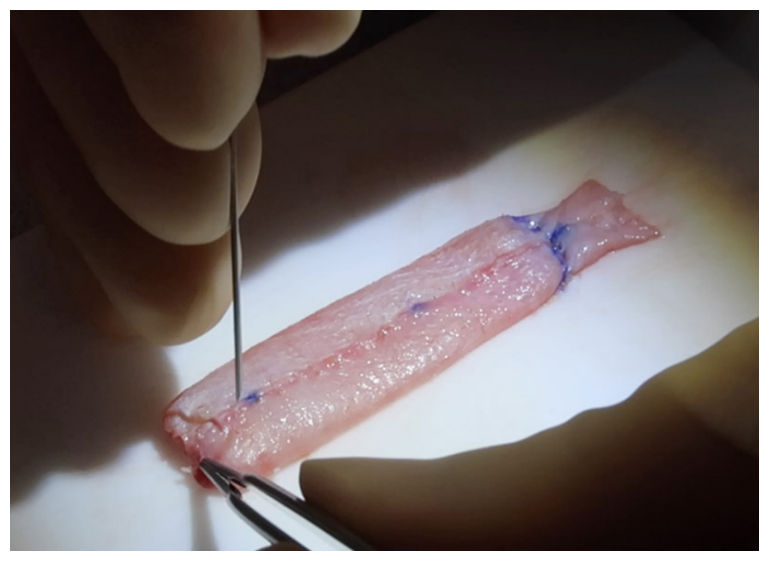

Figure 6. Fenestrations are created throughout the diced cartilage fascia to allow for free effusion of any remaining fluid to promote earlier vascular and fibrous ingrowth

along the supratip break with a 5-0 vicryl, creating a portion of the construct filled with diced cartilage, and a tab of fascia without cartilage used to secure the complex to the supratip and tip complex, as shown in Figures 4 and 5.

A needle is used to create fenestrations throughout the DCF, to allow for free effusion of any remaining fluid within the construct, and to promote quicker fibrous and vascular ingrowth into the graft [Figure 6]. Corset sutures are placed to taper the graft from a cylindrical shape to a more parabolic shape, consistent with the appearance of the desired dorsal aesthetic lines [Figure 7]. These corset sutures may be used to great effect to finely calibrate the proportions and dimensions of the graft.

When the final shape has been achieved, the DCF may be placed again along the dorsum of the nose to evaluate the size and shape one final time prior to placement. Deficiency or excess volume and height may be adjusted by making a small incision along the DCF and removing or adding diced cartilage as deemed necessary.

The DCF is secured along its cephalic and caudal ends, and its body shaped by casting. A percutaneous suture is placed through the marked starting point, and secured to the cephalic end of DCF. In cases of excessively wide skin dissection and a resultant large dorsal pocket - such as in removal of a previous implant or graft, multiple percutaneous sutures may be placed to allow for more secure fixation. Along the caudal aspect of the construct, the fascia is secured to the supratip and over the tip complex. The nasal skin envelope may then be re-draped and the shape of the dorsum evaluated. 


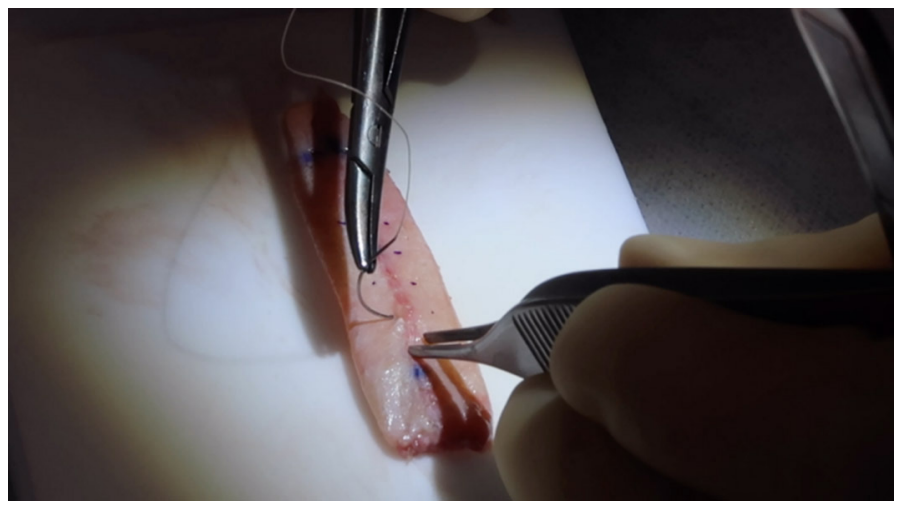

Figure 7. Precise placement of corset sutures allows for the creation of dorsal aesthetic lines
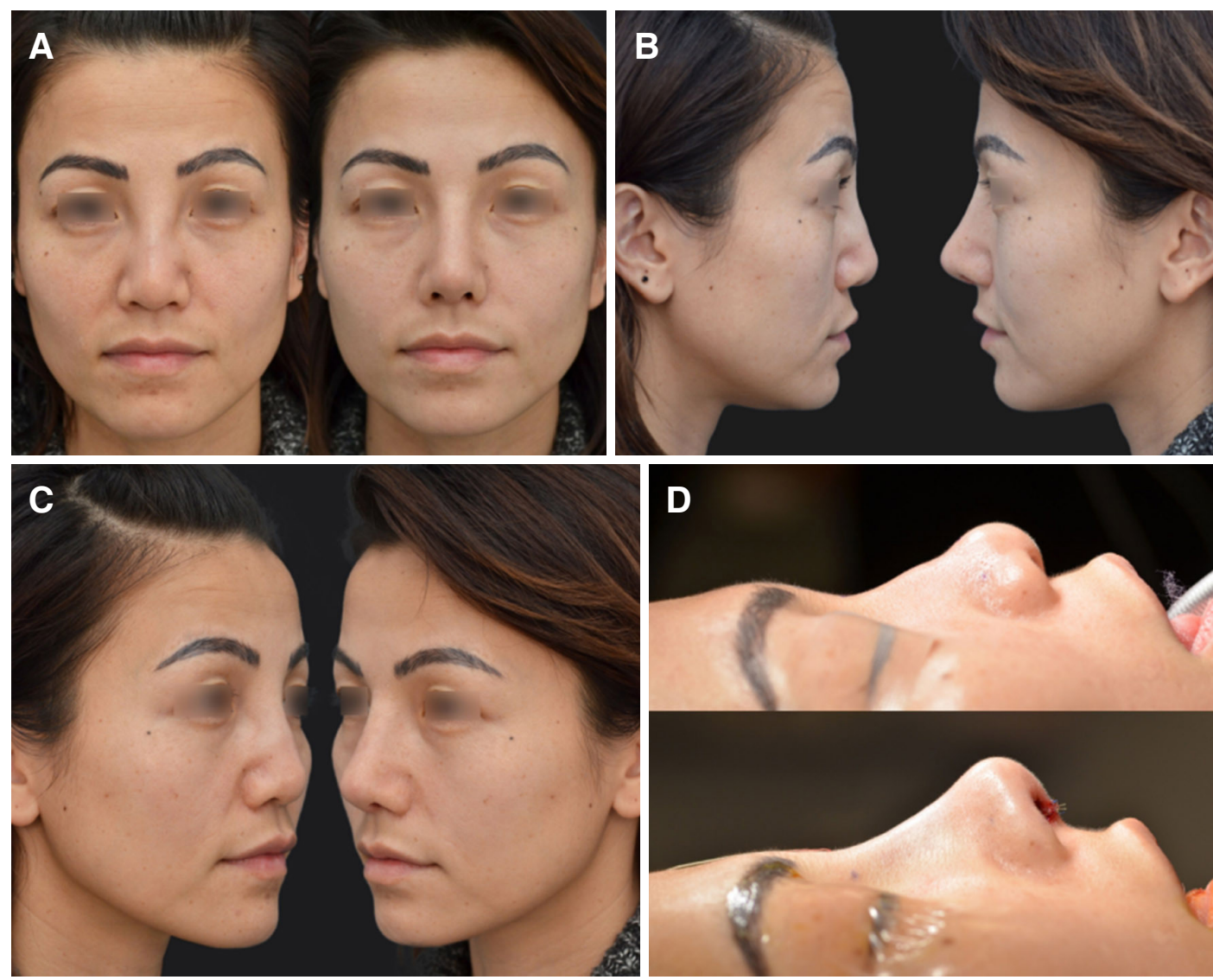

Figure 8. $(A, C)$ Frontal, oblique and $(B, D)$ lateral views of Patient 1 before and 2.5 years after primary rhinoplasty with rib cartilage and diced cartilage fascia

Casting is the last step and is critical for a successful result. The shape and position of the mid-portion of the graft relies heavily on precise molding and contouring of the cast to shape the coagulum of diced cartilage within the DCF. For this reason, casting with a thermoplastic splint is recommended to allow for precise shaping. Once the ideal shape has been obtained, ice-cold water is poured liberally on the cast to lock in the final shape. The cast and percutaneous sutures are removed 1 week post-operatively. Case examples of primary and revision rhinoplasties using the updated dice cartilage technique are demonstrated in Figures $8-10$, respectively. The dorsum will initially be much wider and taller, but the majority of the swelling will resolve in 3-6 months with the final results in 1-2 years. 

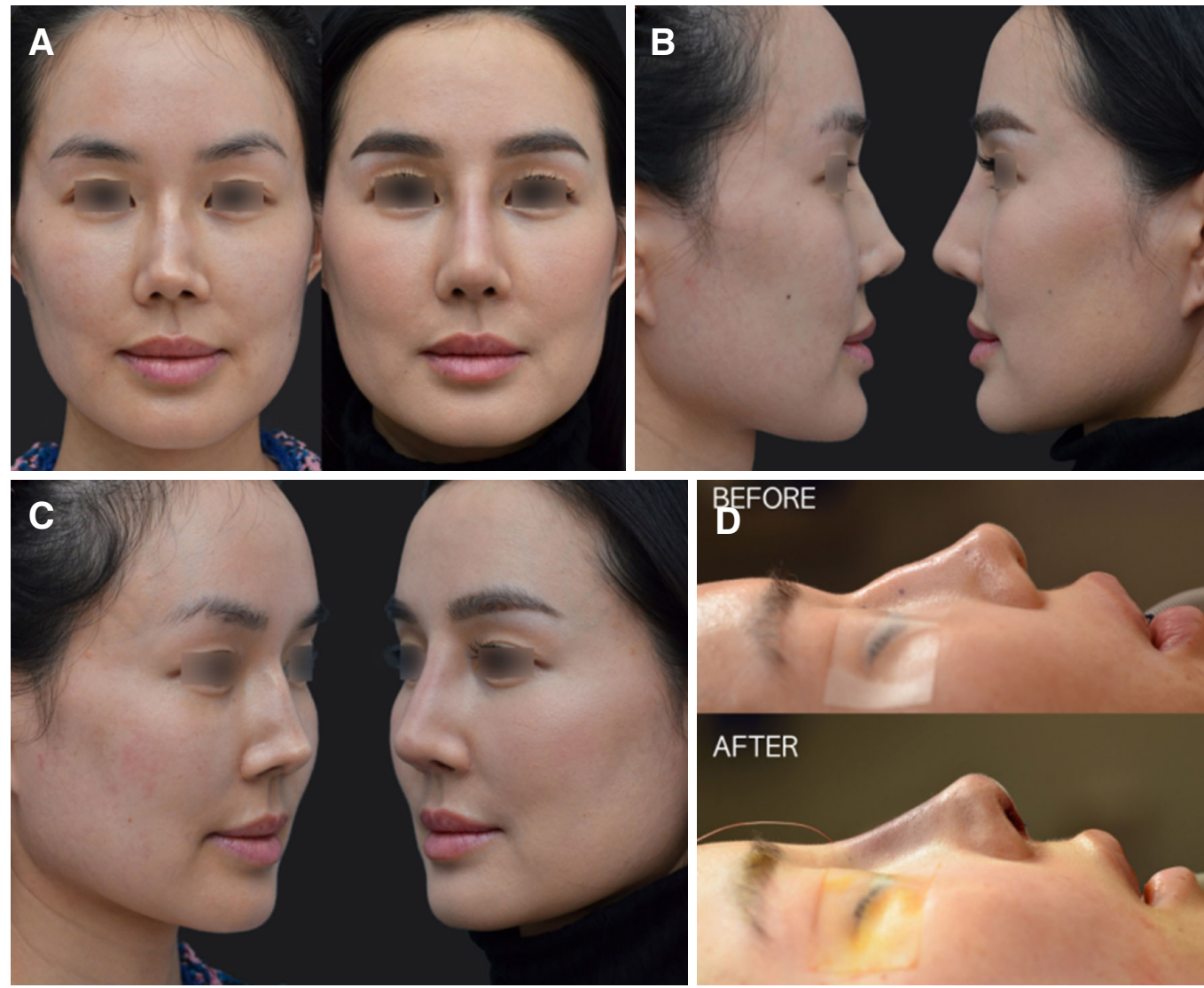

Figure 9. ( $A, C$ ) Frontal, oblique and (B, D) lateral views of Patient 2 before and 8 months after revision rhinoplasty with rib cartilage and diced cartilage fascia. Previous over-aggressive rhinoplasty resulted in low dorsum
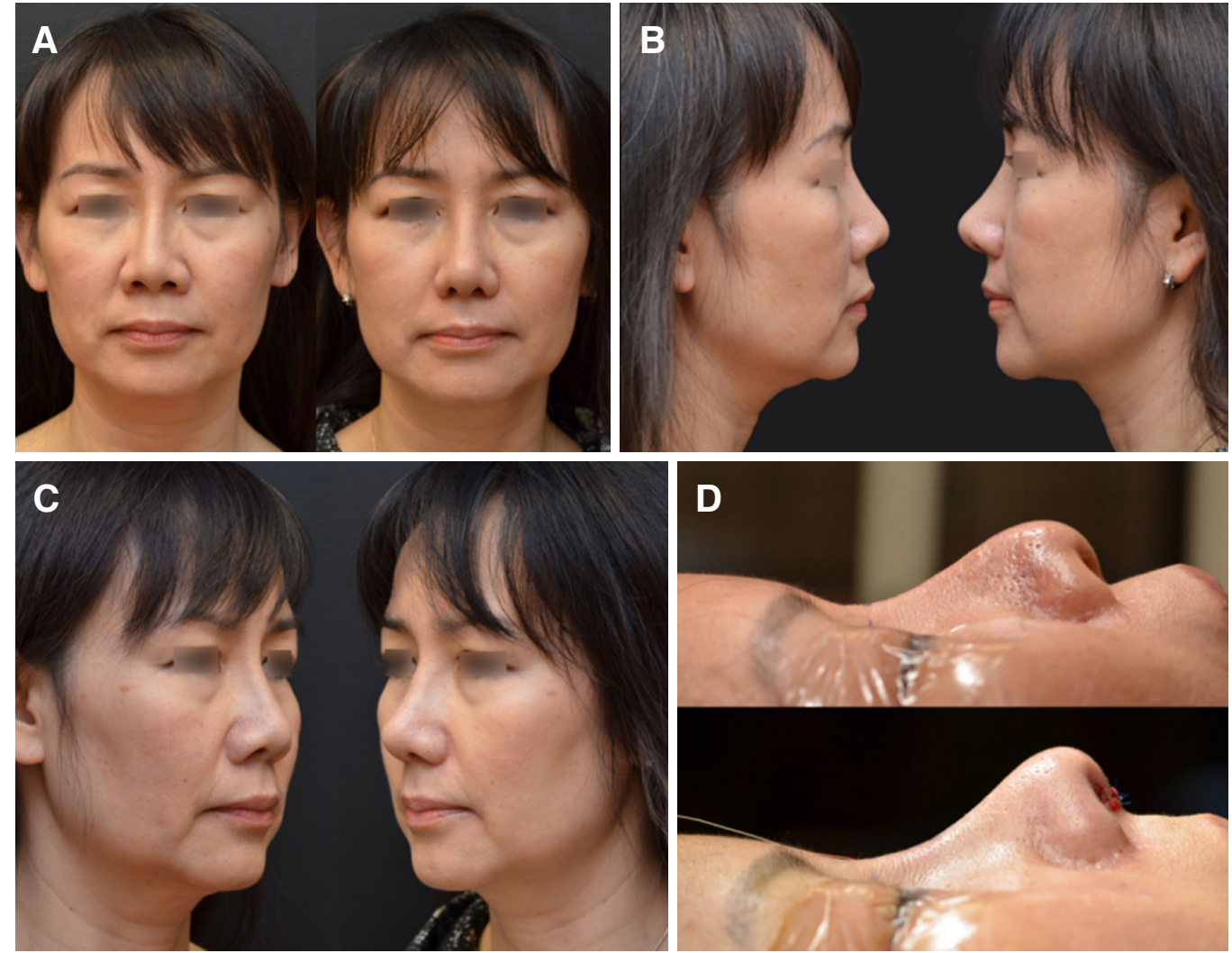

Figure 10. (A, C) Frontal, oblique and (B, D) lateral views of Patient 3 before and 1 year after revision Asian rhinoplasty with rib cartilage and diced cartilage fascia. Previous rhinoplasty with silicone implant 


\section{DISCUSSION}

Given the contemporary focus of minimizing complications and creating a life-long result, many rhinoplasty surgeons have shifted towards exclusively using autologous grafts during dorsal augmentation. Diced cartilage fascia techniques have proven attractive for a number of reasons, including their relative pliability, wide availability of materials needed for the construct, and the perceived forgiving nature with regards to contour irregularities.

Diced cartilage fascia techniques for dorsal augmentation in rhinoplasty and revision rhinoplasty have been variously utilized and described for over half a century. Despite producing satisfactory results in many cases, it has received criticism at times for creating a "sausage-like" appearance or an otherwise unnatural look to the dorsum. Also despite its perception as forgiving, it does have a somewhat large inter-surgeon variance with regards to aesthetic outcomes. The term is broadly descriptive, and there remains a wide-range of ways to execute it.

Contour irregularities remain the most common reason for surgeon and patient dissatisfaction after dorsal augmentation using diced cartilage with fascia. Sub-optimal contours may manifest in the form of convexities and concavities, over or under augmentation, deviation, asymmetries, and unnatural dorsal aesthetic lines. Occasionally, natural variations in nasal skin envelope thickness and sebaceous qualities between the dorsum, supratip, tip, infratip and columella, as well as scarring from previous surgeries, may result in a less than ideal appearance to the nasal starting point, radix, dorsum, supratip break, nasal tip, infratip lobule, and columella. Conservative management of minor contour irregularities with nasal exercises (especially within the first month following surgery), and directed injections of kenalog and 5-fluorouracil, will successfully address many of the irregularities observed in the early post-operative period. Persistent contour irregularities beyond post-operative edema involving coalesced diced cartilage will infrequently warrant revision surgery to address.

This updated diced cartilage fascia technique seeks to eliminate variance and enhance precision to create more predictable and consistently beautiful results. Placing greater emphasis on precision and a more algorithmic approach to constructing the DCF graft may result in even improved outcomes for future patients.

\section{DECLARATIONS}

\section{Authors' contributions}

The authors contributed solely to the article.

\section{Availability of data and materials}

Not applicable.

\section{Financial support and sponsorship}

None.

\section{Conflicts of interest}

All authors declared that there are no conflicts of interest.

\section{Ethical approval and consent to participate}

Written informed consent was obtained for all patients.

\section{Consent for publication}

Written informed consent was obtained for all patient images. 


\section{Copyright}

(C) The Author(s) 2019.

\section{REFERENCES}

1. Vilar-Sancho B. An old story: an ivory nasal implant. Aesthetic Plast Surg 1987;11:157-61.

2. Krause CJ. Augmentation rhinoplasty. Otolaryngol Clin North Am 1975;8:743-52.

3. Wheeler ES, Kawamoto HK, Zarem HA. Bone grafts for nasal reconstruction. Plast Reconstr Surg 1982;69:9-18.

4. Romo T 3rd, Jablonski RD. Nasal reconstruction using split calvarial grafts. Otolaryngol Head Neck Surg 1992;107:622-30.

5. Leaf N. SMAS autografts for the nasal dorsum. Plast Reconstr Surg 1996;97:1249-52.

6. Daniel RK. Rhinoplasty: an atlas of surgical techniques. New York: Springer; 2002. pp. 11-2.

7. Daniel RK, Calvert JW. Diced cartilage grafts in rhinoplasty surgery. Plast Reconstr Surg 2004;113:2156-71.

8. Guerrerosantos J, Trabanino C, Guerrerosantos F. Multifragmented cartilage wrapped with fascia in augmentation rhinoplasty. Plast Reconstr Surg 2006;117:804-12; discussion 13-6.

9. Cerkes N, Basaran K. Diced cartilage grafts wrapped in rectus abdominis fascia for nasal dorsum augmentation. Plast Reconstr Surg 2016;137:43-51.

10. Regnault P. Nasal augmentation in the problem nose. Aesthetic Plast Surg 1987;11:1-5.

11. Khoo BC. Augmentation rhinoplasty in the orientals. Plast Reconstr Surg 1964;34:81-8.

12. Beekhuis GJ. Silastic alar-columellar prosthesis in conjunction with rhinoplasty. Arch Otolaryngol 1982;108:429-32.

13. Wellisz T. Clinical experience with the Medpor porous polyethylene implant. Aesthetic Plast Surg 1993;17:339-44.

14. Godin MS, Waldman SR, Johnson CM Jr. The use of expanded polytetrafluoroethylene (Gore-Tex) in rhinoplasty. A 6-year experience. Arch Otolaryngol Head Neck Surg 1995;121:1131-6.

15. Queen TA, Palmer FR 3rd. Gore-tex for nasal augmentation: a recent series and a review of the literature. Ann Otol Rhinol Laryngol 1995; $104: 850-2$.

16. Adams JS. Grafts and implants in nasal and chin augmentation. A rational approach to material selection. Otolaryngol Clin North Am 1987;20:913-30.

17. Juraha LZ. Experience with alternative material for nasal augmentation. Aesthetic Plast Surg 1992;16:133-40.

18. Gilmore J. Use of vicryl mesh in prevention of postrhinoplasty dorsal irregularities. Ann Plast Surg 1989;22:105-7.

19. Fanous N. Mersilene tip implants in rhinoplasty: a review of 98 cases. Plast Reconstr Surg 1991;87:662-71; discussion 72-3.

20. Gunter JP, Rohrich RJ. Augmentation rhinoplasty: dorsal onlay grafting using shaped autogenous septal cartilage. Plast Reconstr Surg 1990;86:39-45.

21. Peer LA. Diced cartilage grafts: new method for repair of skull defects, mastoid fistula and other deformities. Arch Otolaryngol 1943;38:156-65.

22. Cottle MH. Nasal surgery in children. Eye Ear Nose Throat Mon 1951;30:32-8.

23. Burian F. The plastic surgery atlas. New York: Macmillan; 1968.

24. Erol OO. The Turkish delight: a pliable graft for rhinoplasty. Plast Reconstr Surg 2000;105:2229-41; discussion 42-3.

25. Berghaus A, San Nicolo M, Jacobi C. Use of a fibrinogen-thrombin sponge in rhinoplasty. Hno 2018;66:103-10.

26. Hoehne J, Gubisch W, Kreutzer C, Haack S. Refining the nasal dorsum with free diced cartilage. Facial Plast Surg 2016;32:345-50.

27. Erol OO. Injection of compressed diced cartilage in the correction of secondary and primary rhinoplasty: a new technique with 12 years' experience. Plast Reconstr Surg 2017;140:673e-85.

28. Tasman AJ. Advances in nasal dorsal augmentation with diced cartilage. Curr Opin Otolaryngol Head Neck Surg 2013;21:365-71.

29. Tasman AJ. Dorsal augmentation-diced cartilage techniques: the diced cartilage glue graft. Facial Plast Surg 2017;33:179-88.

30. Kovacevic M, Riedel F, Wurm J, Bran GM. Cartilage scales embedded in fibrin gel. Facial Plast Surg 2017;33:225-32. 